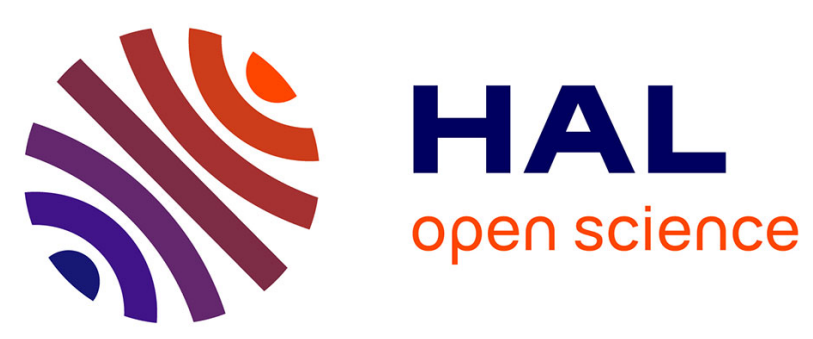

\title{
Femtosecond relaxation processes in nonlinear materials studied with incoherent light
}

T. Kobayashi, T. Hattori, A. Terasaki, K. Kurokawa

\section{To cite this version:}

T. Kobayashi, T. Hattori, A. Terasaki, K. Kurokawa. Femtosecond relaxation processes in nonlinear materials studied with incoherent light. Revue de Physique Appliquée, 1987, 22 (12), pp.1773-1785. 10.1051/rphysap:0198700220120177300 . jpa-00245738

\section{HAL Id: jpa-00245738 https://hal.science/jpa-00245738}

Submitted on 1 Jan 1987

HAL is a multi-disciplinary open access archive for the deposit and dissemination of scientific research documents, whether they are published or not. The documents may come from teaching and research institutions in France or abroad, or from public or private research centers.
L'archive ouverte pluridisciplinaire HAL, est destinée au dépôt et à la diffusion de documents scientifiques de niveau recherche, publiés ou non, émanant des établissements d'enseignement et de recherche français ou étrangers, des laboratoires publics ou privés. 


\title{
Femtosecond relaxation processes in nonlinear materials studied with incoherent light
}

\author{
T. Kobayashi, T. Hattori, A. Terasaki and K. Kurokawa \\ Department of Physics, Faculty of Science, University of Tokyo, 7-3-1 Hongo, Bunkyo-ku, Tokyo 113, Japan
}

(Reçu le 12 juin 1987, révisé le 16 août 1987, accepté le 31 août 1987)

\begin{abstract}
Résumé. - Trois types de mesures rapides utilisant une lumière temporellement incohérente plutôt que des impulsions courtes sont décrites. Le déphasage électronique dans un film de polydiacétylène, le déphasage vibrationnel dans le diméthyl sulfoxyde liquide et la relaxation de l'effet Kerr optique dans le $\mathrm{CS}_{2}$ et dans le nitrobenzène sont observés avec une résolution femtoseconde.
\end{abstract}

\begin{abstract}
Three types of short-time measurements using temporally incoherent light instead of short pulses are described. Electronic dephasing in a polydiacetylene film, vibrational dephasing in dimethylsulfoxide liquid, and relaxation of optical Kerr effect in $\mathrm{CS}_{2}$ and nitrobenzene were observed with femtosecond time resolution.
\end{abstract}

\section{Introduction.}

Increasing number of scientists have been studying the dynamical properties of matter. The development of picosecond and femtosecond spectroscopies has brought much information with higher time resolution. Since the first demonstration of the generation of picosecond light pulses using passively mode-locked ruby laser in 1965 [1], continuous efforts to get shorter pulses have been made, and optical pulses as short as $8 \mathrm{fs}$ were obtained [2a] by the method of pulse compression of the output from a group-velocity-dispersion-compensated collidingpulse mode-locked laser. Very recently the shortest pulse of $6 \mathrm{fs}$ was obtained by the compression method using a prism pair and a grating pair for the compensation of both second- and third-order group velocity dispersion $[2 \mathrm{~b}]$. Time-resolved coherent and conventional spectroscopies have been applied to several systems including semiconductors and dye molecules using ultrashort light pulses with pulse width of a few tens to a hundred femtoseconds. However, there are several difficulties and disadvantages in the study of the ultrafast phenomena using such short pulses : i) laser systems for the generation of ultrashort pulses applicable to spectroscopies are necessarily very expensive and complicated. ii) The wavelengths of laser pulses with pulse width shorter than a few hundred femtoseconds are usually limited in the region around $615-625 \mathrm{~nm}$ because of the lack of appropriate combination of saturable absorber and gain medium, and the tunability of each laser is generally poor (usually less than $10 \mathrm{~nm}$ ). iii) It is difficult to avoid broadening of an ultrashort pulse due to linear and/or nonlinear dispersion in optics such as lenses, filters, and samples because of its broad power spectrum.

Recently a new spectroscopic technique with incoherent light utilizing coherent transient optical effects has been proposed and its applicability to actual material systems was verified experimentally [3-7]. Since ordinary electronic devices do not have subpicosecond or femtosecond time resolution, optical experiments using that time region are usually performed using nonlinear optical phenomena. In these methods, the signal light generated or modulated by nonlinear optical effects is detected for the measurement of response of matter, using the correlation between excitation and probe light beams. In typical experimental systems, an optical pulse is split into two beams, and they are coupled to each other in a sample after passing through variable and fixed optical delay lines, and the intensity of signal or probe light is measured as a function of the delay time. Generally the signal intensity is expressed by the time integration of functions of the field amplitude (or intensity) and the response function of the matter.

In the studies of the dynamics taking place in matter, therefore, the time resolution is expected to be determined by the correlation time instead of the pulse duration. According to this principle, extreme- 
ly high time resolution can be much more easily obtained by using light with a short enough correlation time. The applicability of this principle to ultrafast process studies has been verified for the dephasing time measurement by degenerate fourwave mixing (DFWM) spectroscopy [3-8].

In the first part of this article, we describe the study of dephasing in a polydiacetylene film measured by spatial-parametric-mixing type DFWM (or sometimes called forward DFWM) [9]. Dephasing times in a polydiacetylene (poly-3BCMU) film were resolved for the first time at two wavelengths by DFWM using incoherent light. The measured dephasing times, $50-60$ fs at $648 \mathrm{~nm}$ and $140-160 \mathrm{fs}$ at $582 \mathrm{~nm}$, correspond to excitons in polymer chains associated with the electronic states having different conjugation lengths.

Though transient DFWM spectroscopy, both using coherent short pulses [10] and incoherent cw light sources as mentioned above, is powerful for the study of dynamic properties of matter, it cannot be applied to optically forbidden transitions and the available wavelength range is limited. Dephasing of Raman active vibrational modes in molecules can be observed by so-called transient coherent Raman spectroscopy such as CARS (coherent anti-Stokes Raman scattering) or CSRS (coherent Stokes Raman scattering) [11-13], where a pair of picosecond pulses excites a vibrational system coherently and a second pulse of the higher frequency probes the coherence of the system after a certain delay time. The information about the dephasing dynamics of the system can be obtained by the dependence of the coherent Raman scattering intensity on the delay time.

We studied theoretically a possible application of the principle that the correlation time determines the resolution time, to transient coherent Raman spectroscopy. Theoretical derivation of the delaytime dependence of the coherent Raman intensity, and the experimental demonstration of the determination of the dephasing time of the $2915-\mathrm{cm}^{-1}$ mode in dimethylsulfoxide are described in the second part [14].

In the last part of this article, we describe the time-resolved study of the relaxation times of the third-order susceptibility for the optical Kerr effect in $\mathrm{CS}_{2}$ and nitrobenzene performed by the phaseconjugation-type DFWM using 5-ns pulses from a broad-band laser [15]. In the case of $\mathrm{CS}_{2}$ we determined both subpicosecond (about $0.2 \mathrm{ps)}$ and picosecond (about $2 \mathrm{ps}$ ) relaxation times for the susceptibility-tensor element of $\chi_{x y y x}^{(3)}$ and also for $\chi_{x x x x}^{(3)}$ both at $553 \mathrm{~nm}$ and at $623 \mathrm{~nm}$. The results agree quite well with those obtained utilizing subpicosecond pulses, and the shorter and longer time constants are attributed to the libration and reorientation relaxation, respectively.

\section{Electronic dephasing in a polydiacetylene film} measured by degenerate four-wave mixing.

There has been much interest in the dynamical properties of the excited states of polydiacetylenes (PDAs), which is one of the typical quasi-onedimensional systems. They have been studied experimentally by time-resolved absorption, reflection and emission spectroscopy [16-21]. The excited state lifetime of soluble PDA (3 KAU) was found to be $9 \pm 3$ ps in aqueous solution [16] and that of PTS to be about 2 ps in crystalline phase [17].

Knowledge of the dephasing dynamics of PDAs is of great importance not only to elucidate the properties of excited states and the mechanism of the optical nonlinearity but also for various applications such as optical switching and optical signal processing. DFWM was applied to the dephasing time measurement [22-24], but dephasing times have not been resolved so far. Dennis et al. [22] observed DFWM from two PDA ( $2 d$ and $2 j)$ solutions with $180 \mathrm{ps}$ pulses, but the response times were much shorter than their resolution time. Carter et al. [23] observed DFWM from a PDA (PTS) crystal with 6 ps pulses, and the response time was again shorter than 6 ps. Rao et al. [24] performed similar measurements on a PDA (poly-4BCMU) film with $500 \mathrm{fs}$ pulses, but they could not resolve the dephasing time either.

In this study, we applied DFWM with incoherent light to the measurement of the dephasing times in a film of a PDA, poly[4,6-decadiyne-1,10-diol bis((nbutoxycarbonyl)-methyl) urethane], which is abbreviated as poly-3BCMU. By detecting signals diffracted in two directions simultaneously, we could resolve a dephasing time as short as $30 \mathrm{fs}$. We measured dephasing times of the sample at two wavelengths, $648 \mathrm{~nm}$ and $582 \mathrm{~nm}$, and found that the dephasing of the exciton in a chain of the polymer with a longer conjugation length (at $648 \mathrm{~nm}$ ) is several times faster than that in a chain with a shorter conjugation length (at $582 \mathrm{~nm}$ ). This result is consistent with the difference in the fluorescence efficiencies between the rod-like and coil-like forms of the acetylene-type phase.

2.1 EXPERIMENTAL. - The experimental apparatus used for the dephasing time measurement by the spatial-parametric-mixing type DFWM is shown in figure 1 . The incoherent light source was a broadband dye laser pumped by a $\mathrm{N}_{2}$ laser. In order to obtain very broad-band laser light, the dye laser cavity without any tuning element was constructed with a highly-reflecting aluminum mirror and a glass plate as an output mirror. Rhodamine $6 \mathrm{G}$ and rhodamine 640 were used as laser dyes. The peak wavelengths and the band widths (FWHM) of the power spectra were $582 \mathrm{~nm}$ and $7.7 \mathrm{~nm}$ for the 


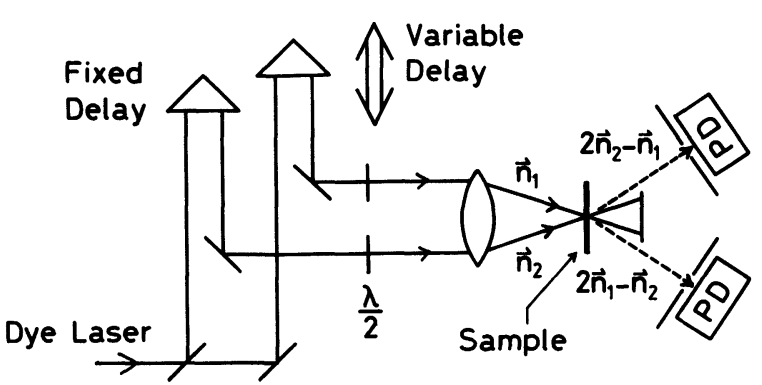

Fig. 1. - Experimental set-up for spatial-parametric-mixing type degenerate four-wave mixing measurement. PD and $\lambda / 2$ stand for a photodiode and a half-wave plate (Fresnel rhomb), respectively. The vectors, $\mathbf{n}_{1}$ and $\mathbf{n}_{2}$, are the unit vectors representing the directions of the two excitation beams.

rhodamine $6 \mathrm{G}$ laser, respectively, and $648 \mathrm{~nm}$ and $8.7 \mathrm{~nm}$ for the rhodamine 640 laser, respectively.

The broad-band dye laser light was linearly polarized and divided into two beams, $\mathbf{n}_{1}$ and $\mathbf{n}_{2}$, and $\mathbf{n}_{2}$ was delayed with respect to $\mathbf{n}_{1}$ by a variable delay line. The polarization planes of the two beams could be rotated independently by the use of half-wave optics composed of two Fresnel rhombs. The pulse energies of the $10 \mathrm{~ns}$ dye laser were about $3 \mu \mathrm{J}$ at the sample position, and the beam diameters of the focused areas on the sample were $40 \mu \mathrm{m}$. Degenerate four-wave mixing signals diffracted in two directions, $2 \mathbf{n}_{2}-\mathbf{n}_{1}$ and $2 \mathbf{n}_{1}-\mathbf{n}_{2}$, were detected simultaneously by photodiodes, as shown in figure 1 [7]. Since in two-beam configuration with equal wavelength there is not exact phase matching, the angle between the two beams should usually be small. However, signal could be observed with the angle as large as $15^{\circ}$ because the sample film was very thin (a few hundred $\mathrm{nm}$ ). Output signals of the photodiodes were processed with sample-and-hold circuits and an A/D converter. The sample was a film of poly-3BCMU on a glass plate cast from chloroform solution. The absorption spectrum of the film is shown in figure 2 .

2.2 Results AND DisCussion. - Figure 3 shows the data which were obtained with the two excitation beams under parallel polarization condition. Background signal due to scattering of the excitation beams (about a few percent of the peak signal intensity) was subtracted from the data. No peak shift or tail was observed. The signal with parallel polarizations was about thirty times more intense than with perpendicular polarizations, and it can be attributed almost exclusively to a thermal grating, which is generated only when mutual coherence between the two excitation beams exists [10].

The contribution of the thermal grating to the DFWM signals is eliminated under perpendicular polarization, and hence we can obtain electronic DFWM signals [7], which are shown in figure 4,

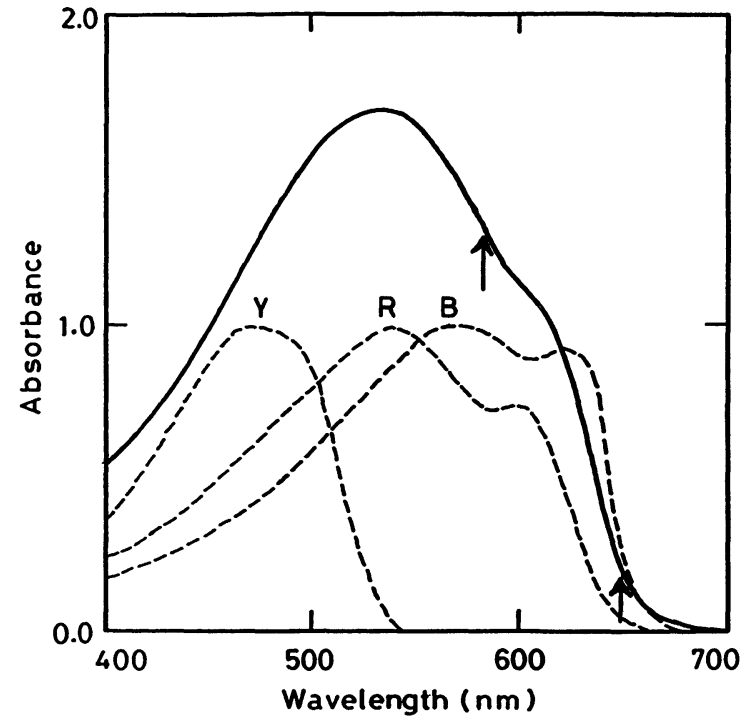

Fig. 2. - Absorption spectrum of the film of poly3BCMU (solid line) with those of three forms in solution from reference [25] (broken lines) which are normalized to the peak values. B, R, and Y denote blue, red, and yellow forms, respectively. Two exciting wavelengths utilized in the present study, $648 \mathrm{~nm}$ and $582 \mathrm{~nm}$, are indicated by arrows.

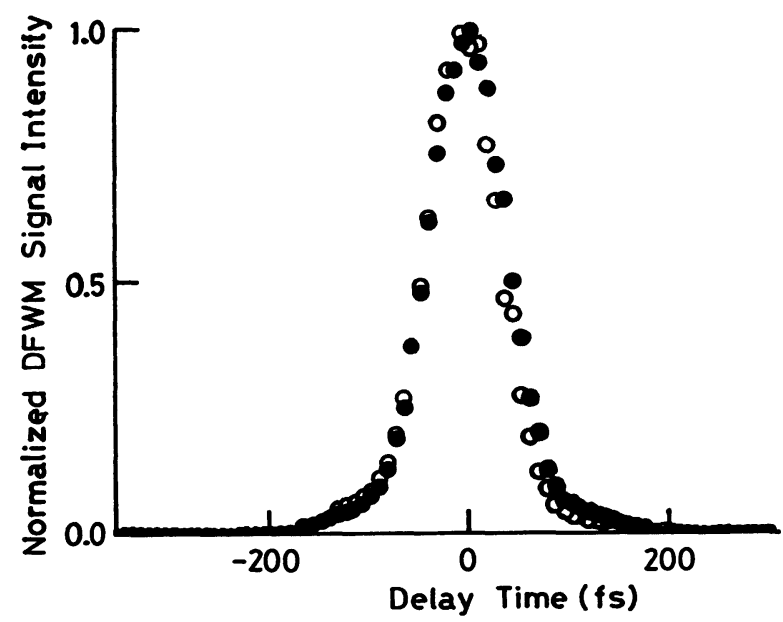

Fig. 3. - DFWM signals obtained with the two excitation beams of polarizations parallel to each other. The wavelength of the excitation light was $648 \mathrm{~nm}$. Open circles show the signal intensity diffracted in the direction $2 \mathbf{n}_{1}-\mathbf{n}_{2}$, and closed circles show that in the direction $2 \mathbf{n}_{2}-\mathbf{n}_{1}$

after the background scattering intensity being subtracted. The peaks of the signal intensities of the two directions are shifted from each other, as shown in figure 4 . Here we define a peak separation as the distance in the delay time between the two peaks of the signal intensities of the two directions. The peak separations were 30 and $90 \mathrm{fs}$ at 648 and $582 \mathrm{~nm}$, respectively. The widths (FWHM) of the signals were $100 \mathrm{fs}$ for $648 \mathrm{~nm}$ and $130 \mathrm{fs}$ for $582 \mathrm{~nm}$, which give the correlation time of the incoherent excitation 

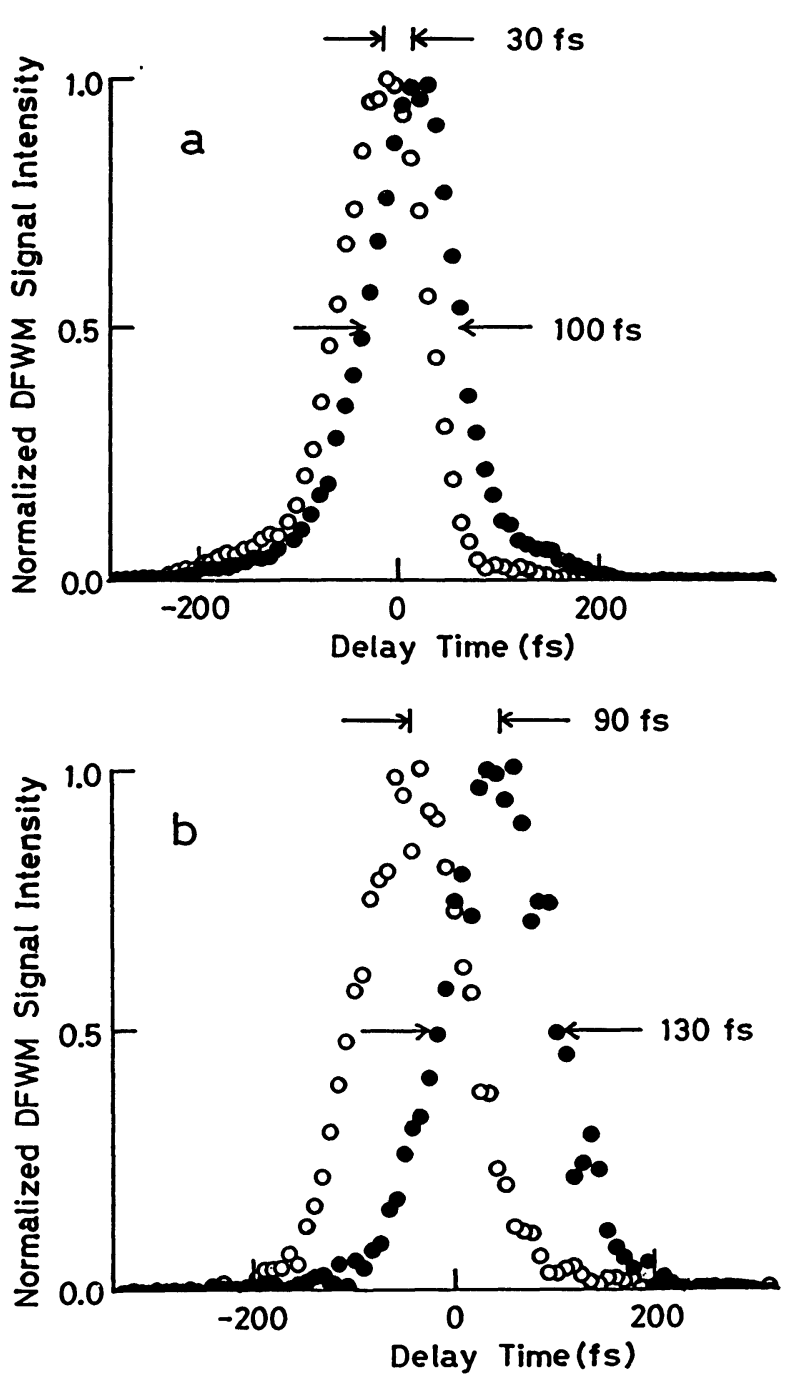

Fig. 4. - DFWM signals with the two excitation beams of polarizations perpendicular to each other. Open circles show the signal intensity diffracted in the direction $2 \mathbf{n}_{1}-\mathbf{n}_{2}$, and closed circles show that in the direction $2 \mathbf{n}_{2}-\mathbf{n}_{1}$. The wavelengths of the excitation light are a) $648 \mathrm{~nm}$ and b) $582 \mathrm{~nm}$.

light at each wavelength with a certain factor according to the signal shape and the definition of correlation time. There is no pronounced asymmetric tails which indicate dephasing times much longer than the correlation time. The tails seen in the data at $648 \mathrm{~nm}$ are the same as those seen in thermal grating signals, and they reflect the shape of the laser spectrum.

Under the condition that the excitation beam is on resonant with a two-level system with large inhomogeneous broadening, the delay-time dependence of the signal intensities is expressed by the following equation [6] :

$$
\begin{aligned}
& I\left(\tau_{\mathrm{d}}\right) \propto \int_{0}^{\infty} \mathrm{d} t \int_{0}^{\infty} \mathrm{d} t^{\prime} G\left(t^{\prime}-t\right) G\left(t-\tau_{\mathrm{d}}\right) \times \\
& \times G^{*}\left(t^{\prime}-\tau_{\mathrm{d}}\right) \exp \left[-2\left(t+t^{\prime}\right) / T_{2}\right] .
\end{aligned}
$$

Here $T_{2}$ is the dephasing time, $\tau_{\mathrm{d}}$ is the delay time, and $G\left(\tau_{\mathrm{d}}\right)$ is the autocorrelation function of the incoherent light field. When the dephasing time is much longer than the correlation time of the light field, the signal decays exponentially at the rate of $4 / T_{2}$. On the other hand, when the dephasing time is comparable with or even slightly shorter than the correlation time, the signal shapes have no prominent tails. This is the case in the present study as presented laser, but the dephasing time can also be obtained from the peak separation of the two signals diffracted in two directions [7, 10]. Using equation (1) and the observed peak separations the dephasing times are calculated to be $30 \mathrm{fs}$ at $648 \mathrm{~nm}$ and $130 \mathrm{fs}$ at $582 \mathrm{~nm}$ by assuming Gaussian autocorrelation functions. The values may change depending on the deviation of the actual material system from the theoretical model, but it is certain that the dephasing at $648 \mathrm{~nm}$ is several times faster than that at $582 \mathrm{~nm}$.

We would like to mention here the effect of the width of the inhomogeneous broadening on the magnitude of the peak separation. Equation (1) is only correct for extremely broad inhomogeneous widths compared with the homogeneous width. In actual materials, the inhomogeneous width is finite, and the effect of the width on the peak separation should be taken into account. For narrower inhomogeneous width the peak separation becomes smaller, and there is no shift for completely homogeneous system. Hence the dephasing time estimated by the observed peak separation may be shorter than the real value. In the present case, the inhomogeneous width can be estimated as $30-50 \mathrm{~nm}$ from the band structure of $0-0$ transition around $600 \mathrm{~nm}$, and the calculated values for the dephasing times are $50-60$ and $140-160 \mathrm{fs}$ at 648 and $582 \mathrm{~nm}$, respectively. Therefore, it can be concluded that the dephasing time for $648 \mathrm{~nm}$ has some ambiguity, but it is considerably shorter than for $582 \mathrm{~nm}$.

The result that the dephasing time at a longer wavelength is shorter than that at a shorter wavelength is contrary to those of previous studies $[7,10]$. A larger peak separation was reported at a longer wavelength than at a shorter wavelength with cresyl fast violet in cellulose, and was explained in terms of the difference in the intramolecular relaxation rate at the two wavelengths [7]. Dephasing time measurements of three dyes, cresyl violet, Nile blue, and oxazine 720 , in polymethylmethacrylate (PMMA) at $15 \mathrm{~K}$ at $620 \mathrm{~nm}$ were also reported [10]. The peak separation for cresyl violet was $60 \mathrm{fs}$, whereas for the other two dyes shifts were shorter than $20 \mathrm{fs}$. The difference in the dephasing times was attributed to that in the excess photon energy of the excitation light from the absorption edge of each sample.

The present sample is a polymeric film which has a quasi-one-dimensional structure and electronic properties different from ordinary dyes $[25,26]$. It is known that poly-3BCMU in solution has three forms 
with different absorption spectra. They are blue (B), red $(\mathrm{R})$, and yellow ( $\mathrm{Y})$ forms [25]. The blue form is considered to have a polymer main chain with rodlike conformation, while $\mathrm{R}$ and $\mathrm{Y}$ forms have main chains with coil-like conformation. These three forms are realized depending on the temperature and the composition of the solvent. The shoulder of the absorbance of the sample in the present study at $620 \mathrm{~nm}(2.0 \mathrm{eV})$ corresponds to the B band, and the peak at $530 \mathrm{~nm}(2.3 \mathrm{eV})$ corresponds to the $\mathrm{R}$ band. They are attributed to the $\pi-\pi^{*}$ exciton transition in each type of the polymer chain. The absorption spectrum of the sample film does not show that it is a mixture of these distinct forms, and it is regarded as a mixture of polymer chains with continuously distributed conjugation lengths between two extreme conformations, a coil-like conformation (with shorter conjugation lengths of $\pi$-electron) and a rod-like conformation (with longer conjugation lengths), which are realized in solution.

In the present experiment, the wavelength of $648 \mathrm{~nm}$ is on the absorption edge of the rod-like form exciton, while that of $582 \mathrm{~nm}$ is also on resonance with the exciton in polymer chains with shorter conjugation lengths. Therefore, from the present results on the dephasing times at the two wavelengths, it can be concluded that the exciton dephasing is several times faster in the rod-like form than in a chain with a shorter conjugation length.

The present result may be explained by the following two mechanisms. One explanation is that excitons are more mobile and the phases of them are changed more often in longer conjugated chains than shorter ones, where excitons do not move over long distances. The other explanation is that exciton levels lie more, closely in longer conjugated chains than in shorter conjugated chains, and therefore, dephasing due to multilevel excitation is faster [10]. We cannot determine which is the case only from the present data. An extended study at other wavelengths and temperatures is in progress.

It has been reported that the fluorescence intensities are suppressed when the solution of poly$3 \mathrm{BCMU}$ is converted from a yellow (coil-like) form to a blue (rod-like) form, and when the solution of PDA (poly-4BCMU) is converted from the coil form to the rod form [25]. It is also reported that only partially polymerized crystal of PDA (PTS) emits fluorescence [27]. Our results are consistent with the explanation $[25,27]$ by which the changes in the fluorescence quantum efficiencies were attributed to the increase in the nonradiative decay rates with exciton delocalization.

\section{Vibrational dephasing time measurement by cohe- rent Stokes Raman scattering.}

The method for the observation of picosecond to femtosecond electronic dephasing dynamics discus- sed in the previous section is extended to the vibrational dephasing time measurement. For the latter experiment a type of nondegenerate four-wave mixing with both coherent and incoherent light is utilized. In the present paper theoretical study is presented first and experimental description follows. It is based on a transient coherent Raman process with three beams (see Fig. 5), two of which are incoherent light from a single broad-band laser, and a delay time between the two is variable. A third beam has a higher frequency than the incoherent light by a vibrational energy in a molecule of interest, and is coherent in the delay-time range of the measurement. The delay-time dependence of coherent Stokes Raman scattering (CSRS) intensity offers the information about the coherence dynamics of the vibrational transition with a resolution time limited by the correlation time of the incoherent light. When the frequency of the third beam is lower than the others, coherent anti-Stokes Raman scattering (CARS) takes place. For the theoretical calculation, a three-level model of molecular system with homogeneous broadening is used, and the delay-time dependence of CSRS intensity was calculated. Dephasing dynamics of the $2915-\mathrm{cm}^{-1}$ mode in dimethylsulfoxide was observed experimentally by the new method using a broad-band nanosecond dye laser, and the obtained dephasing time was found to agree well with that obtained with picosecond pulses. Raman echo experiment with incoherent light is also proposed in the last part of this chapter.

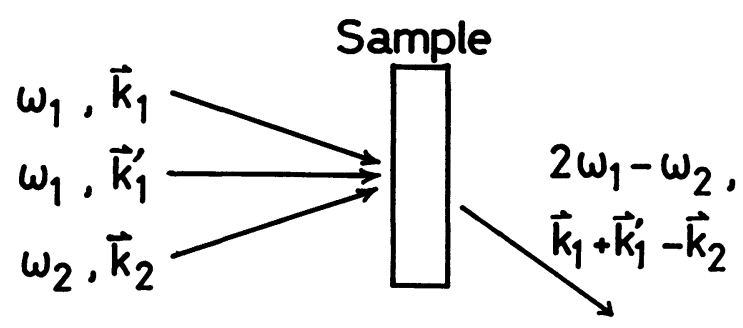

Fig. 5. - Schematic of CSRS experiment for vibrational dephasing measurement.

3.1 DESCRIPTION OF A MODEL AND TIME DEPENDENCE OF SIGNAL INTENSITY. - Until now all reported studies of transient spectroscopy with incoherent light, including photon echo and pump-probe spectroscopy, were concerned with only one broad band (about $100 \mathrm{~cm}^{-1}$ ) within which exists the resonance frequency to the transition of a two-level system. However, there exist various coherent transient phenomena where light beams of two or more different frequencies are concerned, and the time resolution of the transient coherent spectroscopies using these phenomena is also expected to be determined not by the duration of light pulses used but by the correlation time of the radiation field. 
In this section, theoretical expectation values of the coherent Raman signal intensity using incoherent light will be presented with a simple model. Calculation of the time-dependent CSRS intensity will be presented for the correspondence with the experimental study described in the following section, although CARS is substantially the same.

A simple model of a three-level system (see Fig. 6) is usually taken for theoretical considerations of coherent Raman phenomena $[28,29]$. Two vibrational levels $|1\rangle$ and $|2\rangle$ belong to the ground electronic state, whereas level $|3\rangle$ belongs to an electronically excited state. Levels $|1\rangle$ and $|3\rangle$ and levels $|2\rangle$ and $|3\rangle$ are connected with each other by electronic transition dipoles. The energy difference between $|3\rangle$ and $|1\rangle$ is $\hbar \Omega_{3}$, and that between $|2\rangle$ and $|1\rangle$ is $\hbar \Omega_{2}$. In ordinary coherent Raman experiments, this system is placed in radiation field which consists of light beams of two frequencies, the difference between which is resonant with the transition between $|1\rangle$ and $|2\rangle$. The higher frequency is denoted by $\omega_{\mathrm{AS}}$ and the lower by $\omega_{\mathrm{L}}$.
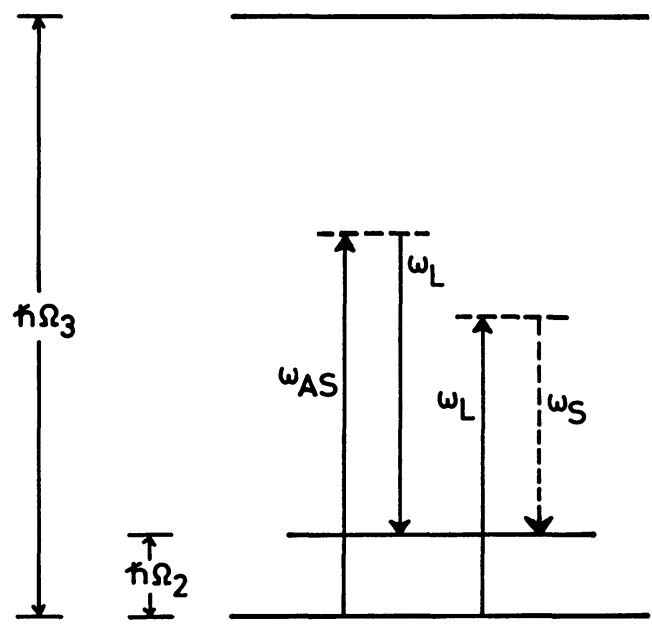

|3>

Fig. 6. - Energy diagram of the model system for vibrational dephasing experiment.

CSRS is a third-order effect, and the intensity is proportional to the light intensity of frequency $\omega_{\mathrm{AS}}$ and to the squared light intensity of frequency $\omega_{\mathrm{L}}$. In usual CSRS (or CARS) experiments two beams are used, but for the purpose of time-resolved measurement, a three-beam configuration was applied [11], where two beams of frequency $\omega_{L}$ and one beam of $\omega_{\mathrm{AS}}$ are used, and the three waves are mixed to generate a wave of frequency $2 \omega_{\mathrm{L}}-\omega_{\mathrm{As}}$.

The electric field is given as

$$
\begin{aligned}
E(\mathbf{r}, t) & =E_{\mathrm{AS}}(t) \exp \left[i\left(\mathbf{k}_{\mathrm{AS}} \mathbf{r}-\omega_{\mathrm{AS}} t\right)\right] \\
& +E_{\mathrm{L} 1}(t) \exp \left[\mathrm{i}\left(\mathbf{k}_{\mathrm{L} 1} \mathbf{r}-\omega_{\mathrm{L}} t\right)\right] \\
& +E_{\mathrm{L} 2}(t) \exp \left[i\left(\mathbf{k}_{\mathrm{L} 2} \mathbf{r}-\omega_{\mathrm{L}} t\right)\right]+\text { c.c. },
\end{aligned}
$$

where c.c. stands for complex conjugates of the preceding terms and $E_{\mathrm{AS}}(t), E_{\mathrm{L} 1}(t)$, and $E_{\mathrm{L} 2}(t)$ are functions of $t$ slowly varying compared to the optical frequencies. In three-beam experiments, light of frequency $\omega_{\mathrm{S}}=2 \omega_{\mathrm{L}}-\omega_{\mathrm{AS}}$ and with wave vector $\mathbf{k}_{\mathrm{S}}=\mathbf{k}_{\mathrm{L} 1}+\mathbf{k}_{\mathrm{L} 2}-\mathbf{k}_{\mathrm{AS}}$ is detected. This can be performed easily by separating spatially the signal from the other light beams because of the directionality of the signal and the laser beams.

The polarization with frequency $\omega_{S}$ and wave vector $\mathbf{k}_{\mathrm{S}}$ is derived by a perturbation method under the following conditions assumed: i) light frequencies are tuned exactly to the vibrational energy. ii) Both of the light of frequencies $\omega_{\mathrm{AS}}$ and $\omega_{\mathrm{L}}$ are off-resonance with electronic transitions. iii) The broadening of the relevant energy level of the molecular system is homogeneous. Under these conditions, a third-order polarization $P^{(3)}\left(\mathbf{k}_{\mathrm{S}}, \omega_{\mathrm{S}}\right)$ is given by

$$
\begin{aligned}
P^{(3)}\left(\mathbf{k}_{\mathrm{S}}, \omega_{\mathrm{S}}\right)=C & \cdot \exp \left[i\left(\mathbf{k}_{\mathrm{S}} \mathbf{r}-\omega_{\mathrm{S}} t\right)\right] \\
\times \int_{-\infty}^{t} \mathrm{~d} t^{\prime} \exp [- & \left.\left(t-t^{\prime}\right) / T_{2}\right] \times\left[E_{\mathrm{L} 1}(t) E_{\mathrm{L} 2}\left(t^{\prime}\right)\right. \\
+ & \left.E_{\mathrm{L} 2}(t) E_{\mathrm{L} 1}\left(t^{\prime}\right)\right] E_{\mathrm{AS}}^{*}\left(t^{\prime}\right)
\end{aligned}
$$

where $T_{2}$ is the dephasing time of the vibrational transition, and $C$ is a time-independent proportionality coefficient. This expression has two terms, in which $E_{\mathrm{L} 1}$ and $E_{\mathrm{L} 2}$ are exchanged with each other.

In the present CSRS experiments using incoherent light, two incoherent light beams of central frequency $\omega_{\mathrm{L}}$ are obtained by splitting a beam from a broad-band dye laser. One of the two beams is delayed to the other with a delay time $t_{\mathrm{d}}$, and the light of frequency $\omega_{\mathrm{AS}}$ is assumed to be coherent in the delay time region of observation.

When one assumes that the correlation time of the incoherent light is much shorter than the dephasing time, and that the stochastic property of the incoherent light is expressed in terms of a Gaussian random process, one can derive a simple expression for the signal intensity as

$$
\begin{aligned}
& I\left(\tau_{\mathrm{d}}\right)=1+G\left(\tau_{\mathrm{d}}\right) \\
& +\left(2 \tau_{\mathrm{c}} / T_{2}\right) \exp \left(-2\left|\tau_{\mathrm{d}}\right| / T_{2}\right) .
\end{aligned}
$$

Here $G(t)$ is the autocorrelation function of the incoherent light field amplitude normalized to unity at its peak, and the correlation time $\tau_{\mathrm{c}}$ is defined by

$$
\tau_{\mathrm{c}}=\int_{0}^{\infty} G(t) \mathrm{d} t
$$

In the above expression (4), the fraction due to the third term of the signal intensity at zero delay time is approximately proportional to the ratio of the correlation time to the dephasing time. Therefore, 
for the dephasing time determination with high precision, incoherent light with an appropriate correlation time must be used, since low intensity signal cannot be distinguished from the background due to inevitable noises from various sources.

3.2 EXPERIMENT AND RESULTS. - CSRS signal by the symmetric $\mathrm{CH}$-stretching vibration of dimethylsulfoxide (DMSO) with a wavenumber $2915 \mathrm{~cm}^{-1}$ was measured by the apparatus shown in figure 7 . Coherent light source was the second harmonic $(532 \mathrm{~nm})$ of a Q-switched Nd : YAG laser operated at $8 \mathrm{~Hz}$. Since the correlation time of this light source is estimated to be about $30 \mathrm{ps,} \mathrm{it} \mathrm{can} \mathrm{be}$ regarded safely as coherent for the time period shorter than $10 \mathrm{ps}$. The main part (about $90 \%$ intensity) of this beam was split off and used to pump a broad-band dye laser. By changing the laser dye concentration, the oscillation wavelength was tuned to $630 \mathrm{~nm}$ which is resonant with the Raman mode of DMSO. The spectral width (FWHM) of the laser light was $7 \mathrm{~nm}$, which corresponds to a correlation time of about $100 \mathrm{fs}$.

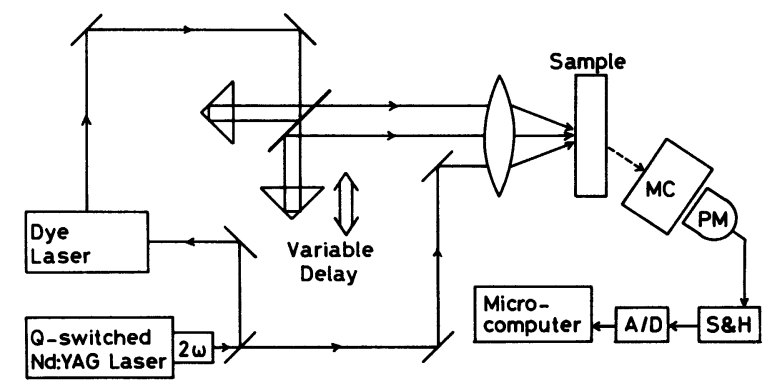

Fig. 7. - Experimental set-up for CSRS measurement using incoherent light and coherent light. $\mathrm{MC}$ is the monochromator ; PM, the photomultiplier.

The dye laser beam was split into two with nearly equal intensity using a beam splitter. Both of them were made parallel again by the same beam splitter after passing through delay lines. The length of one of the delay lines was varied by a stepping motor. The three beams were focused in DMSO in a $10-\mathrm{mm}$ sample cell by a lens of $20-\mathrm{cm}$ focal length. To eliminate the scattered laser light, an iris with $5-\mathrm{mm}$ diameter, a $10-\mathrm{cm}$ monochromator, and color filters were placed between the sample and the photomultiplier. To make the angles among the three beams large, nonplanar (folded BOXCARS) configuration [30] was employed, where the two dye laser beams lie on the horizontal plane and the YAG laser beam was slightly out of that plane.

In figure 8 , the CSRS signal intensity is plotted as a function of the delay time in a semilogarithmic scale after the background is subtracted, where the signal intensity is normalized to the background

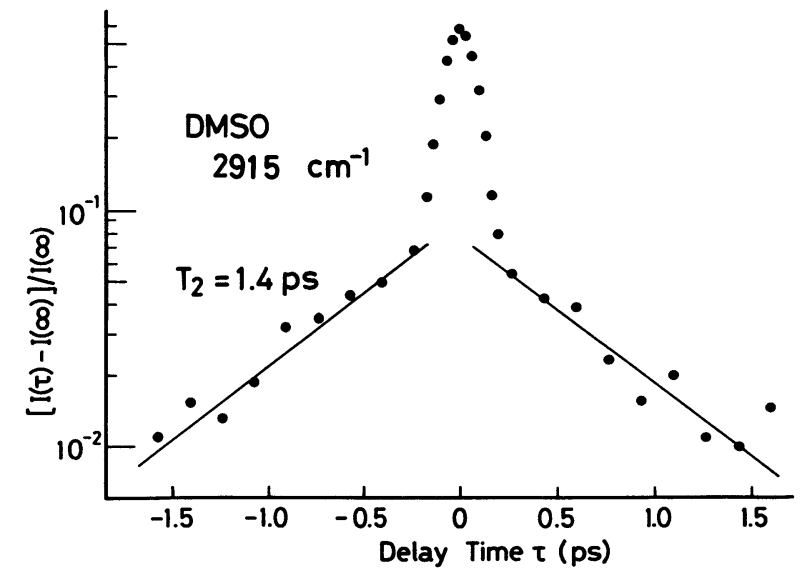

Fig. 8. - The delay-time dependence of the CSRS intensity normalized by the background intensity. The background intensity has been subtracted.

intensity. From the slope of the trailing part, the vibrational dephasing time $T_{2}$ is found to be $1.4 \mathrm{ps}$ in agreement with the value obtained with picosecond pulses [12]. The intensity ratio between the peak and the tail extrapolated to $t_{\mathrm{d}}=0$ is about seven, which is also in good agreement with the theoretically expected ratio for the values of $T_{2}(1.4 \mathrm{ps})$ and the correlation time (100 fs) determined above.

\subsection{PROPOSAL OF RAMAN ECHO EXPERIMENT WITH} INCOHERENT LIGHT. - Since CSRS or CARS does not include a rephasing process, which takes place in photon echoes, the obtained value of the dephasing time has some ambiguities when the system is inhomogeneously broadened [31, 32]. Raman echo [28] can provide the information about the dephasing dynamics without the ambiguities [29]. However, it is a higher-order (seventh-order) process, and the detection of the signal is very difficult. Raman echo experiments have been carried out in solids [33], gases [34, 35], and liquid nitrogen [36], but not yet in liquids at room temperature.

The following type of Raman echo experiment may be feasible with incoherent light (see Fig. 9).

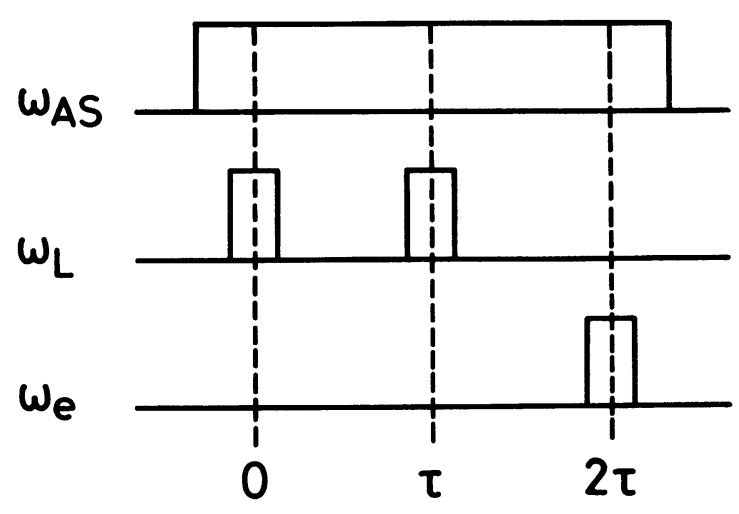

Fig. 9. - Schematic of Raman echo experiment. 
The molecules are brought in coherent superposition of the ground state and a vibrationally excited state by light of two frequencies, $\omega_{\mathrm{AS}}$ and $\omega_{\mathrm{L}}$ at $t=0$. The difference between these frequencies is matched to the vibrational transition. Rephasing is introduced by light of the same pair of the frequencies at $t=\tau$. The macroscopic coherence is recovered, if the vibrational transition is inhomogeneously broadened, at $2 \tau$, and it is probed by the light of frequency $\omega_{\mathrm{AS}}$, and the emitted light of frequency $\omega_{\mathrm{c}}=2 \omega_{\mathrm{AS}}-\omega_{\mathrm{L}}$ is detected. The two pulses of frequency $\omega_{L}$ in the figure are replaced in the actual experiment by two incoherent light beams with a delay of $\tau$ obtained from the same light source.

Since in this experimental scheme the probing pulse is not a short pulse but light with a constant intensity, the emitted signal light may include a portion due to the free Raman susceptibility decay (the Raman analogue of the free induction decay). However, the dephasing time can be estimated fairly well by the echo decay rate and the background intensity even in this case. The expression for the signal intensity as a function of the delay time is completely the same as the one for the dephasing measurement by two-beam DFWM with incoherent light whether the system has either homogeneous or inhomogeneous broadening [3]. Of course, the incoherent light can be used as the probe pulse for the detection of only the echo signal. For this purpose a third incoherent beam which is delayed by $\tau$ with respect to the second one is used as the probe light, and the emitted light of frequency $2 \omega_{L}-\omega_{A S}$ is detected.

\section{Molecular dynamics of Kerr liquids studied by optical phase conjugation.}

We extend the incoherent method to another type of the degenerate four-wave mixing, i.e., the phase conjugation in a Kerr medium. Liquid $\mathrm{CS}_{2}$ is one of the most familiar and simplest optical Kerr media with a large nonresonant nonlinear refractive index $n_{2}$ [37]. In 1975, a direct time-resolved measurement using a picosecond laser revealed that the relaxation time of optically induced birefringence in $\mathrm{CS}_{2}$ is $2.1 \mathrm{ps}$ [38]. The recent development of the generation technique of subpicosecond and femtosecond optical pulses made easier the relaxation-time measurement of the optical Kerr effect with higher time resolution. Using a time-resolved interferometric technique with 70 -fs pulses at $619.5 \mathrm{~nm}$, Halbout and Tang observed both a 2.00 -ps response and a 0.33-ps response with standard deviations of 17 fs [39]. Greene and Farrow measured two relaxation times, $2.16 \pm 0.1$ and $0.24 \pm 0.02$ ps, utilizing a biased Kerr shutter with $150-\mathrm{fs}$ pulses at $620 \mathrm{~nm}$ [40]. Using a usual Kerr shutter with 200-fs pulses, Etchepare et al. observed a response of $1.4 \pm 0.1$ and $0.20 \pm 0.05 \mathrm{ps}$. Pulses with two wavelengths were used in their measurement : $615 \mathrm{~nm}$ for the pump pulse and $650 \mathrm{~nm}$ for the probe pulse [41]. In all the three measurements subpicosecond (0.2-0.3 ps) and picosecond (1.4-2.2 ps) relaxations with almost the same corresponding time constants were observed. Also in the frequency domain, Trebino et al. measured the relaxation times of four nonzero third-order-susceptibility elements for the optical Kerr effect in $\mathrm{CS}_{2}$ using a tunable-laser-induced-grating technique with an excitation wavelength of $575 \mathrm{~nm}$ and a probe wavelength of $570 \mathrm{~nm}$. They obtained, for example, two relaxation times of $2.49 \pm 0.5 \mathrm{ps}$ and $0.21 \pm 0.04 \mathrm{ps}$ for $\chi_{x x x x}^{(3)}[42]$.

The picosecond relaxation in $\mathrm{CS}_{2}$ is considered to be associated with orientational randomization by rotational diffusion of molecules. On the other hand, the mechanism of the subpicosecond response is not well explained. Several mechanisms have been proposed as its possible physical origin : the damped librational motion, the time-dependent behaviour resulting from collisions, and the short-time behaviour associated with the rotational diffusion of molecules possessing a large anisotropic polarizability $[39,40,42,43]$.

Liquid nitrobenzene is another well-known optical Kerr medium. A subpicosecond relaxation time of about 150 fs was obtained by Tang and Halbout by an interferometric technique [39]. Etchepare et al. observed subpicosecond ( $<0.2 \mathrm{ps)}$ and picosecond ( 4 ps and $42 \mathrm{ps)} \mathrm{relaxation} \mathrm{times} \mathrm{with} \mathrm{the} \mathrm{Kerr}$ shutter of nitrobenzene [41].

In this article we report for the first time the timeresolved optical phase conjugation in Kerr liquids, $\mathrm{CS}_{2}$ and nitrobenzene, using incoherent light. The subpicosecond (about $0.2 \mathrm{ps}$ ) and the picosecond (about $2 \mathrm{ps)} \mathrm{relaxation} \mathrm{times} \mathrm{were} \mathrm{observed} \mathrm{in}$ $\mathrm{CS}_{2}$. The details of the experimental procedure and results obtained by the time-resolved phase conjugation in $\mathrm{CS}_{2}$ and nitrobenzene are presented in the following.

4.1 EXPERIMENTAL. - The experimental set-up used for the measurements of the phase-conjugate signal in both Kerr liquids is shown in figure 10 . The incoherent light source was similar to that used in the experiment on PDA except that the excitation source was an excimer laser. Rhodamine 560 ( Rh $560)$ in methanol or rhodamine $640(\mathrm{Rh} \mathrm{640)}$ in ethanol was used as the laser dye both in the oscillator and the amplifier. The peak wavelength was $553 \mathrm{~nm}$ and the width (FWHM) of the spectrum was $5.5 \mathrm{~nm}$ for Rh 560, and they were $623 \mathrm{~nm}$ and $5.0 \mathrm{~nm}$ for $\mathrm{Rh} 640$. The duration of the output pulses was $5 \mathrm{~ns}$, which was much longer than any other time scales treated in this study. The pulse does not have fine structures which exhibit fast intensity change takes place. 


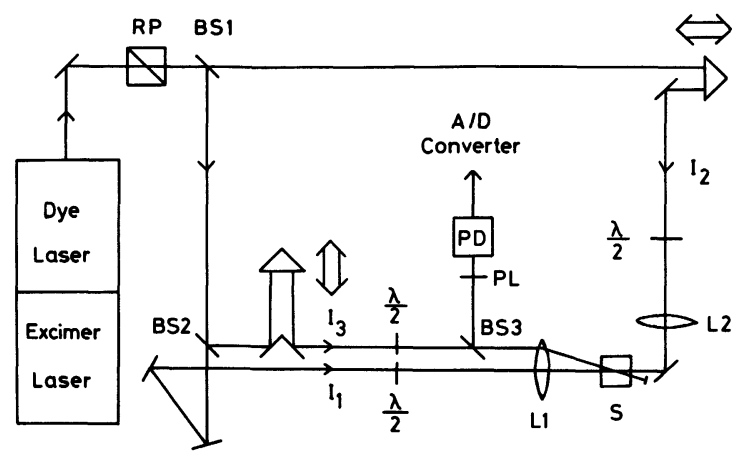

Fig. 10. - Experimental set-up for the phase-conjugation type degenerate four-wave mixing using incoherent light. $\mathrm{BS} 1,2$ is the beam splitter ; BS3, the glass plate ; $\mathrm{L} 1,2$, the lens ; PD, the photodiode ; PL, the polarizer; RP, the Rochon prism ; $\mathrm{S}$, the sample ; $\lambda / 2$, the half-wave retarder.

The dye laser beam was passed through a Rochon prism to obtain a linearly polarized beam, which was split into three beams $I_{1}, I_{2}$, and $I_{3}$ by beam splitters BS1 and BS2. After passing through delay lines beams $I_{3}$ and $I_{2}$ were focused by lenses L1 $(f=70 \mathrm{~mm})$ and $\mathrm{L} 2(f=100 \mathrm{~mm})$, respectively, in the sample. Lengths of the delay lines were varied with stepping motors. The interaction length of the three beams was about $1 \mathrm{~mm}$, which was much longer than the coherence length of the incoherent light. To avoid the ambiguity of the delay time the counterpropagating beam $I_{2}$ was delayed or advanced by $0.2 \mathrm{~ns}$ to the other two beams, $I_{1}$ and $I_{3}$. This was much longer than the correlation time and much shorter than the duration of the pulse, and hence $I_{2}$ had no correlation with $I_{1}$ and $I_{3}$ because the correlation time of the dye laser light was in subpicosecond region. The signal intensity was measured as a function of the delay time $(\tau)$ of beam $I_{1}$ with respect to beam $I_{3}$. Beam $I_{1}$ was made parallel with beam $I_{3}$ and was focused in the $1-\mathrm{cm}$ sample cell by lens L1, while $I_{1}$ and $I_{2}$ were exactly counterpropagating to each other. The peak intensities of beams $I_{1}, I_{2}$, and $I_{3}$ were about 200, 400, and $300 \mathrm{MW} / \mathrm{cm}^{2}$, respectively at the focal point of the Rh 560 laser. They were about 70, 200, and $100 \mathrm{MW} / \mathrm{cm}^{2}$ in the case of the Rh 640 laser. Polarization planes of the three beams can be independently rotated with three half-wave retarders, each of which is composed of coupled Fresnel rhombs. Measurements were performed in the following two polarization configurations :

Configuration I: $I_{1}$ and $I_{2}$, are horizontally polarized and $I_{3}$ is vertically polarized.

Configuration II : $I_{1}, I_{2}$, and $I_{3}$ are all vertically polarized. The angle between beams $I_{1}$ and $I_{3}$ was $4^{\circ}$ in the sample cell. The phase-conjugate signal was split by a glass plate BS3 and detected with a photodiode after passing through an analyser PL.
Output intensity of the photodiode coupled with a sample-and-hold circuit was digitized and transferred to a microcomputer. The excimer laser and all the circuits for detection were synchronized and controlled by a synchronizing circuit and the microcomputer.

4.2 Results AND Discussion. - The semilogarithmic intensity of the normalized phase-conjugate signal in $\mathrm{CS}_{2}$ obtained with $553 \mathrm{~nm}$ laser is shown in figure 11. Beam $I_{2}$ was delayed by about $0.2 \mathrm{~ns}$ with respect to the other two beams. The polarizations of three beams were configuration $I$, that is, $I_{1}$ and $I_{2}$ were horizontally polarized and $I_{3}$ was vertically polarized. Figure 11 also shows a semilogarithmic plot of the normalized signal with configuration II in a reference sample, which is a solution of 4diethylamino-4'-nitrostilbene (DEANS) in $\mathrm{CS}_{2}$ with a concentration of $1 \times 10^{-3}$ mole/l. The absorbance of the reference sample was 0.08 at $553 \mathrm{~nm}$. Since the signal intensity in the reference sample is mainly due to the thermal grating, and it is more than two orders of magnitude larger than that in neat $\mathrm{CS}_{2}$, the effect of the signal due to the nonlinearity of $\mathrm{CS}_{2}$ itself is negligible. As shown in figure 11 the signal obtained for the reference sample is symmetric with respect to the decay time and its FWHM is

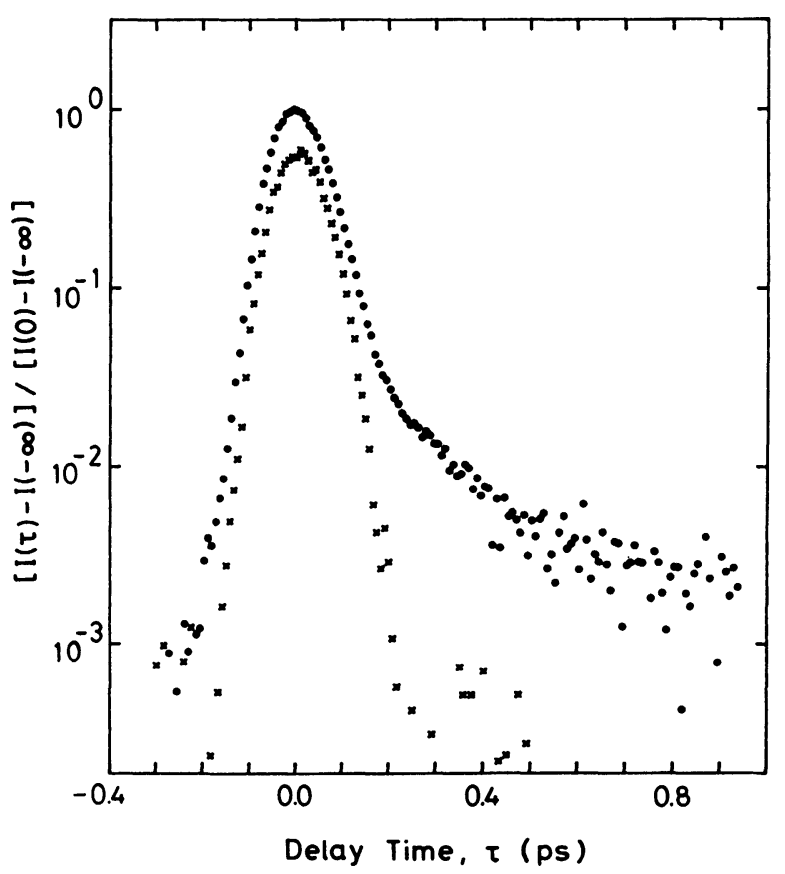

Fig. 11. - Normalized signal intensity as a function of delay time $\tau$ of $I_{1}$ to $I_{3}$. (•) $\mathrm{CS}_{2}$ in configuration $I$, where, $I_{3}$ is vertically polarized and both $I_{1}$ and $I_{2}$ are horizontally polarized. $(\times)$ reference sample (DEANS in $\mathrm{CS}_{2}$ ) in configuration II, where $I_{1}, I_{2}$, and $I_{3}$ are all vertically polarized. The wavelength is $553 \mathrm{~nm}$. Plot of the normalized signal intensity in reference sample is slightly shifted downward. 
0.12 ps. Since $I_{2}$ has no correlation with the other two, the observed signal in the reference sample is considered to be due to a thermal grating produced by the interference between beams $I_{1}$ and $I_{3}$. The observed signal, therefore, gives a correlation profile $|G(\tau)|^{2}$ of the incident incoherent light, where $G(\tau)$ is the autocorrelation of the incoherent field.

On the other hand, the signal in $\mathrm{CS}_{2}$ is clearly asymmetric and consists of three components with different decay times. The signal shape was independent of the scan direction of the delay time. The delay-time dependence of the signal in the case when the beam $I_{2}$ was advanced by about $0.2 \mathrm{~ns}$ to the other two was the same as in figure 11. In figure 11 the fastest decay component among the three is very intense and its time dependence coincides with the reference signal, that is, with the correlation profile $|G(\tau)|^{2}$. In order to determine the longest decay time, measurement in a wider delay-time range was performed, the result being shown in figure 12 . The

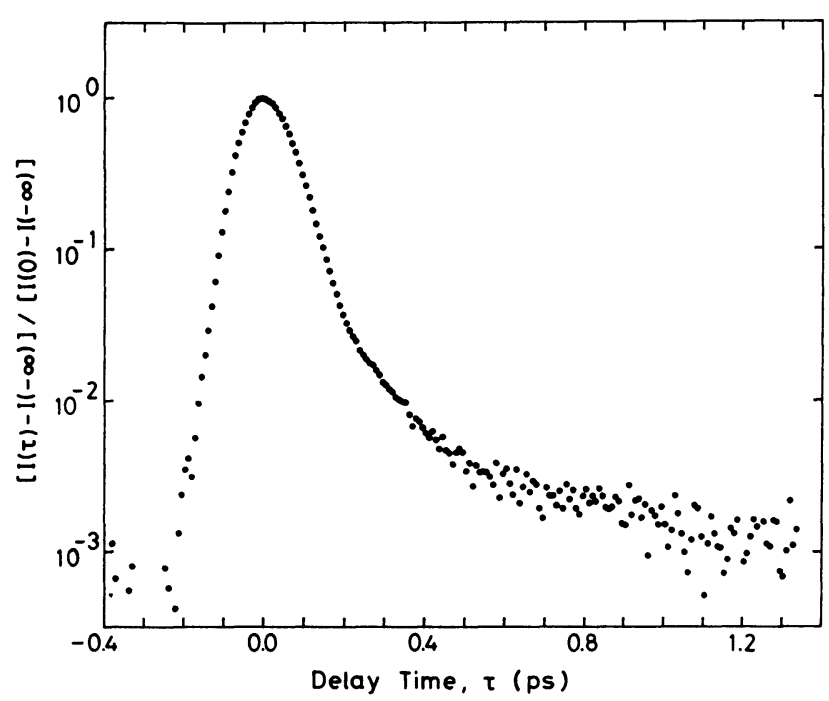

Fig. 12. - Normalized signal intensity as a function of delay time in $\mathrm{CS}_{2}$ at $553 \mathrm{~nm}$. Polarization is configuration $I$.

time constant $\tau_{1}$ was obtained as $0.9_{-0.1}^{+0.2}$ ps. By subtracting the slowest component, the time constant $\tau_{\mathrm{s}}$ of the middle component was obtained as $88 \pm 8 \mathrm{fs}$. Twice these obtained values, that is, $2 \tau_{1}=1.8_{-0.2}^{+0.4} \mathrm{ps}$ and $2 \tau_{\mathrm{s}}=0.18 \pm 0.02 \mathrm{ps}$, agree within experimental error with the long and short time constants, respectively, obtained by ultrashort pulses or by the frequency-domain measurements as mentioned above. Signal intensities were also measured with configuration II at this wavelength, $553 \mathrm{~nm}$, and the two time constants obtained with the data agree with the result of configuration I within experimental error.

Figure 13 shows a semilogarithmic plot of the signal intensity in $\mathrm{CS}_{2}$ of configuration I at $623 \mathrm{~nm}$.

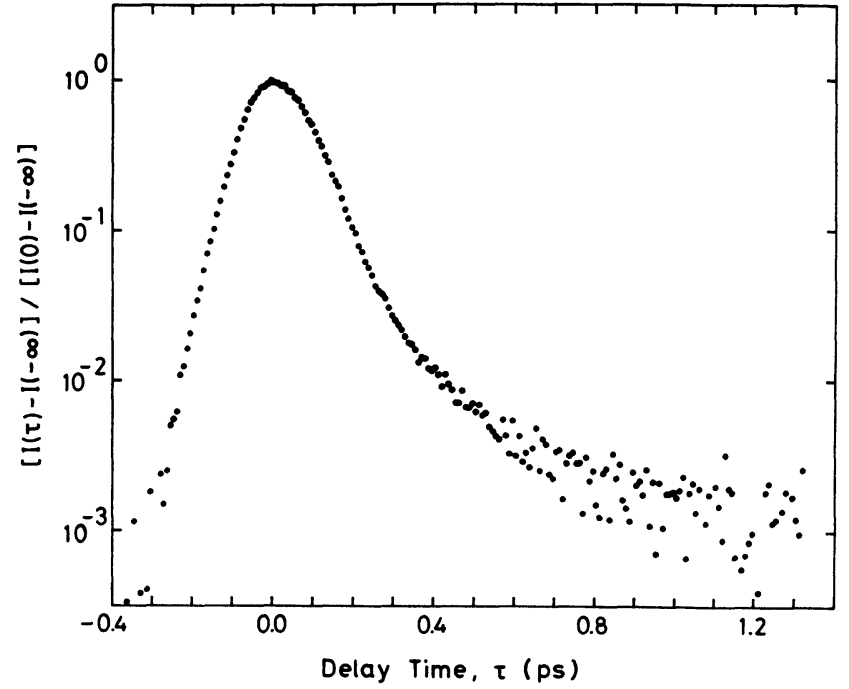

Fig. 13. - Normalized signal intensity as a function of delay time in $\mathrm{CS}_{2}$ at $623 \mathrm{~nm}$. Polarization is configuration I.

The time constants $\tau_{1}$ and $\tau_{\mathrm{s}}$ were obtained as $0.8_{-0.2}^{+0.4} \mathrm{ps}$ and $100 \pm 15 \mathrm{fs}$, respectively. These value agree within experimental errors with those at $553 \mathrm{~nm}$. Two time constants $\tau_{1}$ and $\tau_{\mathrm{s}}$ of configuration II were also obtained at $623 \mathrm{~nm}$ and agree within experimental errors with respective values of configuration I.

We now derive the expression for the delay-time dependence of the intensity of the phase-conjugate signal assuming that the response of Kerr effect is single exponential with a decay-time constant $T$ :

$$
\begin{aligned}
P_{i}^{(3)}(t)= & E_{j}(t) \int_{-\infty}^{t} \mathrm{~d} t^{\prime} r_{i j k l}(0) \times \\
& \times \exp \left[-\left(t-t^{\prime}\right) / T\right] E_{k}\left(t^{\prime}\right) E_{l}\left(t^{\prime}\right),
\end{aligned}
$$

and the decay-time constant $T$ is assumed to be much larger than the correlation time $\tau_{\mathrm{c}}$. The effect of ultrafast (e.g. electronic) response will be mentioned later. Furthermore, we assume the electric field of the incoherent light can be expressed in terms of a stationary complex Gaussian random process [44], and the autocorrelation function of the field decays exponentially :

$$
G(\tau)=\exp \left(-|\tau| / \tau_{\mathrm{c}}\right)
$$

The incoherent light utilized in experiments may not have the characteristic expressed in equation (7), but the signal intensity calculated under the assumption is expected to offer a good approximate value in the delay-time region longer than $\tau_{c}$. Furthermore, we neglect the two-photon resonance contribution to the nonlinear susceptibility.

Using these relations we can obtain the delay-time dependence of the signal intensity as

$$
I_{\mathrm{s}}(\tau) \propto T^{2} \exp \left(-2|\tau| / \tau_{\mathrm{c}}\right)+B+T \tau_{\mathrm{c}}
$$


with

$$
B=\left\{\begin{array}{l}
\frac{\tau_{\mathrm{c}}^{2}}{2} \exp \left(-2|\tau| / \tau_{\mathrm{c}}\right), \quad \tau \leqslant 0 \\
\frac{3 \tau_{\mathrm{c}}^{2}}{2} \exp (-2 \tau / T)-\tau_{\mathrm{c}}\left(\tau_{\mathrm{c}}+\tau\right) \\
\times \exp \left(-2 \tau / \tau_{\mathrm{c}}\right), \quad \tau>0
\end{array}\right.
$$

The first term of equation (8) forms a spike at $\tau=0$ in $I_{\mathrm{s}}(\tau)$. We denote this term as a «spike » term. The second term of equation (8) decays with a time constant of $T / 2$ for long delay time $\tau>\tau_{\mathrm{c}}$. Therefore, this term provides direct information on the relaxation time which is much shorter than the pulse duration. We denote this term as a «signal » term. Since this term has a sharp rise near $|\tau| \sim \tau_{c}$, the time resolution is determined by the correlation time $\tau_{c}$. The third term in equation (8) appears as a background which is independent of the delay time. We denote this term as a « background » term. From equations (8) and (9) relative contributions of the « spike », the «signal», and the «background» terms estimated at $\tau=0$ are $T^{2}, \tau_{c}^{2} / 2$, and $T \tau_{c}$, respectively. Therefore, the first component in the observed signal is considered to be due to the « spike » term. The effect described by the « spike» term is the same one known as the coherence effect involved in the time-resolved optical Kerr effect [45].

The middle and the slowest decay components in the observed signals shown in figures 11,12 , and 13 are considered to be due to the "signal " term. In the delay-time range where the delay time $\tau$ is much longer than the correlation time $\tau_{c}$ the «signal » term decays with the time constant of $T / 2$. Thus, from the measured decay-time constants $\tau_{1}=$ $0.9_{-0.1}^{+0.2} \mathrm{ps}$ and $\tau_{\mathrm{s}}=88 \pm 8 \mathrm{fs}$, relaxation times of the third-order nonlinear polarization in $\mathrm{CS}_{2}$ are obtained to be $1.8_{-0.2}^{+0.4} \mathrm{ps}$ and $0.18 \pm 0.02 \mathrm{ps}$. As previously mentioned, these values agree well with the two time constants determined by the measurements using ultrashort optical pulses.

If we assume the third-order nonlinear polarization is purely electronic, that is, $r_{x y y x}(t)=$ $\sigma_{x y y x} \delta(t)$, then $I_{\mathrm{s}}(-\infty)$ becomes a half of $I_{\mathrm{s}}(0)$. In the present measurements in the configuration $I$, $I_{\mathrm{s}}(-\infty)$ was about $3 \%$ of $I_{\mathrm{s}}(0)$. Therefore, the nuclear contribution is considered to play a major role in $r_{x y y x}$. This result is consistent with the previous study using ultrashort pulses $[40,46]$.

Figure 14 shows semilogarithmic plots of the signal at $553 \mathrm{~nm}$ in nitrobenzene, with the beam $I_{2}$ being delayed by about $0.2 \mathrm{~ns}$ with respect to the others in configuration I. Signal was also observed in configuration II, and the both results coincide with each other and are symmetric and composed of only one component, and no apparent asymmetric component could be observed. The signal intensity of con- figuration II was about thirty times larger than that of configuration I. Therefore, the configuration II signal is attributed mainly to a thermal grating and gives a correlation profile $|G(\tau)|^{2}$ of the incident incoherent light. Thus, the observed signal of configuration $I$ is due to the «spike " term mentioned above. Since the large ratio of $T^{2}: \tau_{c}^{2}$ due to a large rotational relaxation time in nitrobenzene [4 ps and 42 ps (Ref. [41])] than that in $\mathrm{CS}_{2}$ and because of a shorter relaxation time $[39,41]$ than the correlation time, no asymmetric component corresponding to the «signal » term could be observed in nitrobenzene.

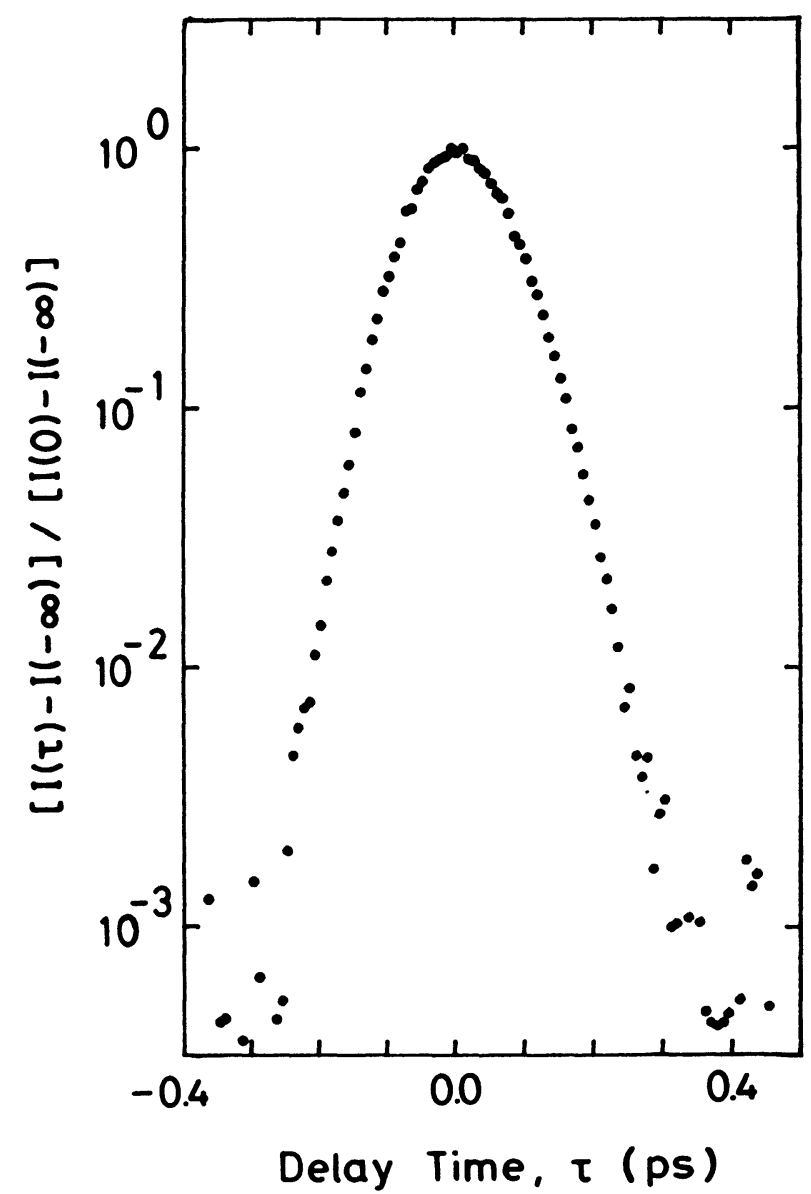

Fig. 14. - Normalized signal intensity as a function of delay time in nitrobenzene at $553 \mathrm{~nm}$. The polarizations of three beams are configuration $\mathrm{I}$.

In summary, we made time-resolved study of subpicosecond-picosecond molecular dynamics in $\mathrm{CS}_{2}$ and nitrobenzene by phase-conjugation type degenerate four-wave mixing using incoherent light. The observed signals with $\mathrm{CS}_{2}$ both at $553 \mathrm{~nm}$ and at $623 \mathrm{~nm}$ consist of three decay components, two of which are characterized by exponential decays. The fastest decaying component of the three coincides 
with the correlation profile $|G(\tau)|^{2}$ of the incident incoherent light. This component is considered as due to the same one as the coherence effect in the time-resolved optical Kerr effect. The middle and the slowest decay components provide direct information on the relaxation times in $\mathrm{CS}_{2}$. The two relaxation times associated with these components agree with the two time constants measured by the use of ultrashort optical pulses. In the case of nitrobenzene the observed signal is symmetric and composed of mainly only one component, which corresponds to the fastest decaying component of the signal observed in $\mathrm{CS}_{2}$. It is considered that because of a longer rotational relaxation time than that in $\mathrm{CS}_{2}$, and because of a shorter relaxation time than the correlation time of the incoherent light, no asymmetric component which provides information about the relaxation time could be observed in nitrobenzene.

\section{Summary.}

We have reported here three types of ultrashorttime measurements using incoherent light, that is, studies of electronic dephasing, vibrational dephasing and Kerr effect dynamics. This technique is effective in these studies and also in other measurements such as the determination of population relaxations of excited states, and the potential applicability is quite extensive because of the simplicity and the tunability of the lasers to be used in this method.

\section{Acknowledgments.}

We wish to thank Professor T. Kotaka for providing us with poly-3BCMU. This work was supported partly by a Grant-in-Aid for Special Distinguished Research (56222005) from the Ministry of Education, Science and Culture of Japan.

\section{References}

[1] Mocker, H. W., Collins, R. J., Appl. Phys. Lett. 7 (1965) 270.

[2] a) Knox, W. H., Fork, R. L., Downer, M. C., Stolen, R. H., Shank, C. V., Appl. Phys. Lett. 46 (1985) 1120.

b) Brito-Cruz, C. H., Fork, R. L., Shank, C. V., MD1 in Technical Digest of Conference on Lasers and Electro-optics, April 27-May 1, 1987, Baltimore, Maryland, USA.

[3] Morita, N., Yajima, T., Phys. Rev. A 30 (1984) 2525.

[4] Asaka, S., Nakatsuka, H., Fujiwara, N., MatsuOKA, M., Phys. Rev. A 29 (1984) 2286.

[5] Beach, R., Hartmann, S. R., Phys. Rev. Lett. 53 (1984) 663.

[6] NaKatsuka, H., Tomita, M., Fujiwara, M., ASAKa, S., Opt. Commun. 52 (1984) 150.

[7] Fujiwara, M., Kuroda, R., Nakatsuka, H., $J$. Opt. Soc. Am. B 2 (1985) 1634.

[8] Meech, S. R., Hoff, A. J., Wiersma, D. A., Chem. Phys. Lett. 121 (1985) 287.

[9] Hattori, T., Kobayashi, T., Chem. Phys. Lett. 133 (1987) 230.

[10] Weiner, A. M., De Silvestri, S., Ippen, E. P., J. Opt. Soc. Am. B 2 (1985) 654.

[11] Laubereau, A., Kaiser, W., Rev. Mod. Phys. 50 (1978) 607.

[12] George, S. M., Auwester, H., Harris, C. B., J. Chem. Phys. 73 (1980) 5573.

[13] George, S. M., Harris, A. L., Berg, M. and Harris, C. B., J. Chem. Phys. 80 (1984) 83.

[14] Hattori, T., Terasaki, A., Kobayashi, T., Phys. Rev. A 35 (1987) 715.

[15] Kurokawa, K., Hattori, T., Kobayashi, T., Phys. Rev. A 36 (1987) 1298.

[16] Koshihara, S., Kobayashi, T., Uchikı, H., Kotaka, T., Ohnuma, H., Chem. Phys. Lett. 114 (1985) 446.
[17] Carter, G. M., Hryniewicz, J. V., Thakur, M. K., Chen, Y. J., Meyler, S. E., Appl. Phys. Lett. 49 (1986) 998.

[18] Kobayashi, T., Iwai, J., Yoshizawa, M., Chem. Phys. Lett. 112 (1984) 360.

[19] Kobayashi, T., Ikeda, H., Tsuneyuki, S., Chem. Phys. Lett. 116 (1985) 515.

[20] Orenstein, J., Etemad, S., Baker, G. L., J. Phys. $C 17$ (1984) L297.

[21] Robins, L., Orenstein, J., Superfine, R., Phys. Rev. Lett. 56 (1986) 1850.

[22] Dennis, W. M., Blau, W., Bradley, D. J., Appl. Phys. Lett. 47 (1985) 200.

[23] Carter, G. M., Thakur, M. K., Chen, Y. J., Hryniewicz, J. V., Appl. Phys. Lett. 47 (1985) 457.

[24] Rao, D. N., Chopra, P., Ghoshal, S. K., SwiatKIEwicz, J., Prasad, P. N., J. Chem. Phys. 84 (1986) 7049.

[25] Kanetake, T., Tokura, Y., Koda, T., Kotaka, T., Ohnuma, H., J. Phys. Soc. Jpn. 54 (1985) 4014.

[26] Chance, R. R., Patel, G. N., Witt, J. D., J. Chem. Phys. 71 (1979) 206.

[27] Sixl, H., Warta, R., Chem. Phys. Lett. 116 (1985) 307.

[28] Hartmann, S. R., IEEE J. Quantum Electron. QE4 (1968) 802.

[29] Loring, R. F., Mukamel, S., J. Chem. Phys. 83 (1985) 2116.

[30] Shirley, J. A., Hall, R. J., Eckbreth, A. C., Opt. Lett. 5 (1980) 380.

[31] Zinth, W., Palland, H.-J., Laubereau, A., Kaiser, W., Appl. Phys. B 26 (1981) 77.

[32] George, S. M., Harris, C. B., Phys. Rev. A 28 (1983) 863.

[33] Hu, P., Geschwind, S., Jedju, T. M., Phys. Rev. Lett. 37 (1976) 1357. 
[34] Leung, K. P., Mossberg, T. W., Hartmann, S. R., Phys. Rev. A 25 (1982) 3097.

[35] Brückner, V., Bente, E. A. J. M., LangelaAr, J., BebelaAr, D., van Voorst, J. D. W., Opt. Commun. 51 (1984) 49.

[36] VAN VOORST, J. D. W., BRANDT, D., VAN Hensbergen, B. L., in Technical Digest of Topical Meeting on Ultrafast Phenomena (1986).

[37] Chiao, R. Y., Garmire, E., Townes, C. H., Phys. Rev. Lett. 13 (1964) 479.

[38] Ippen, E. P., Shank, C. V., Appl. Phys. Lett. 26 (1975) 92.

[39] Halbout, J. M., Tang, C. L., Appl. Phys. Lett. 40 (1982) 765 ;

Tang, C. L., Halbout, J. M., in Picosecond Phenomena III Eds K. B. Eisenthal, R. W. Hochstrasser, W. Kaiser, and A. Laubereau (Springer, Berlin) 1982, p. 212.

[40] Greene, B. I., Farrow, R. C., J. Chem. Phys. 77 (1982) 4779 ;

Greene, B. I., FARrow, R. C., in Picosecond Phenomena III Eds K. B. Eisenthal, R. W.
Hochstrasser, W. Kaiser, and A. Laubereau (Springer, Berlin) 1982, p. 209.

[41] Etchepare, J., Grillon, G., Astier, R., Martin, J. L., BRUNEAU, C., ANTONETTI, A., in Picosecond Phenomena III Eds K. B. Eisenthal, R. W. Hochstrasser, W. Kaiser and A. Laubereau (Springer, Berlin) 1982, p. 217 ;

Etchepare, J., Grillon, G., ANTONETti, A., Chem. Phys. Lett. 107 (1984) 489.

[42] Trebino, R., Barker, C. E., Siegman, A. E., IEEE J. Quantum Electron. QE-22 (1986) 1413.

[43] GolomboK, M., Kenney-Wallace, G. A., in $U l$ trafast Phenomena $I V$ Eds D. H. Auston and K. B. Eisenthal (Springer, Berlin) 1984, p. 383.

[44] Goodman, J. W., Statistical Optics (Wiley, New York) 1985.

[45] Oudar, J. L., IEEE J. Quantum Electron. QE-19 (1983) 713.

[46] Etchepare, J., Grillon, G., Thomazeau, I., Migus, A., Antonetti, A., J. Opt. Soc. Am. B 2 (1985) 649. 